\title{
Conbercept Versus Conbercept Combined with Retinal Photocoagulation for Macular Edema Secondary to Branch Retinal Vein Occlusion
}

Gengmin Tong ( $\nabla$ fkstrer@163.com )

Dongyang People's Hospital https://orcid.org/0000-0002-3754-6491

Yishan Hu

Dongyang People's Hospital

Dawei Wang

Dongyang People's Hospital

Yanhua Jin

Dongyang People's Hospital

Research article

Keywords: injection of conbercept (IVC), branch retinal vein occlusion (BRVO), central macular thickness (CMT), best corrected visual acuity (BCVA)

Posted Date: March 10th, 2021

DOl: https://doi.org/10.21203/rs.3.rs-294143/v1

License: (9) This work is licensed under a Creative Commons Attribution 4.0 International License. Read Full License 


\section{Abstract}

Background: To evaluate the efficacy of intravitreal injection of conbercept (IVC) with or without laser photocoagulation for recurrent macular edema secondary to branch retinal vein occlusion (BRVO).

Methods: 82 patients (82 eyes) with recurrent macular edema secondary to BRVO were collected. The central macular thickness (CMT) and best corrected visual acuity (BCVA) were recorded.

Results: The BCVA in both groups was significantly superior to that before treatment $(P<0.05)$. Compared with initial values, CMT was reduced significantly in both groups $(P<0.05)$. But at 1 month to 6 month after treatment, there are no significant difference was observed between the two groups $(P>0.05)$. In the combined therapy group for Hemispheric Retinal Vein Occlusion, we observed a lower number of reinjections during follow-up.

Conclusion: Conbercept with or without retinal photocoagulation can effectively improve the visual acuity and reduce the CMT.

\section{Background}

Retinal vein occlusion (RVO) is a common cause of visual loss among adults throughout the world, the secondary macular edema (ME) can cause serious visual impairment $[1,2]$. The prevalence rate of branch retinal vein occlusion (BRVO) is greater than central retinal vein occlusion (CRVO). Macular edema and neovascularization are the main complications of BRVO, the treatment of BRVO is directed at secondary complications of BRVO that affect vision [3]. At present, intravitreal anti-vascular endothelial growth factor (VEGF) agents is first-line therapy for macular edema[4]. Anti-VEGF therapy, including intravitreal ranibizumab, aflibercept, bevacizumab and conbercept. Conbercept (also known for Lumitin, or KH903, Kang Hong Biotech Co, Ltd, Sichuan, China) is a recombinant fusion protein[5]. It is suitable for the treatment of visual impairment caused by age-related macular degeneration and choroidal neovascularization.Currently, conbercept is only available in China. Some studies have confirmed that as a result of special Fab fragment, conbercept has a more powerful binding affinity for VEGF than ranibizumab[6, 7]. A few studies have confirmed that intravitreal conbercept injection (IVC) can resolve ME secondary to BRVO[8-10]. But many patients need repeated injections, which increases the financial burden of them. In addition, grid laser photocoagulation is another choice can reduce vascular leakage and alleviate ME secondary to BRVO[11]. But grid laser photocoagulation has a risk of visual impairment. Since both laser photocoagulation and conbercept can effectively treat macular edema secondary to BRVO. Therefore, after treatment with conbercept, whether it is necessary for laser photocoagulation is worth discussing.

This study carried out comparative analysis of intravitreal injection of conbercept and conbercept combined with retinal photocoagulation, to evaluate whether laser photocoagulation combined with conbercept is more beneficial to ME secondary to BRVO. 


\section{Methods}

\section{Trial design}

We retrospectively examined consecutive non-randomized patients who underwent intravitreal conbercept or intravitreal conbercept combine with laser photocoagulation for BRVO at Dongyang people's Hospital, Zhejiang, Republic of China. The study was conducted in accordance with the Declarations of Helsinki and informed consent was obtained from all the enrolled participants. The study was approved by the Ethics Committee of Dongyang people's Hospital.

\section{Participants}

82 eyes of 82 patients with recurrent ME secondary to BRVO were enrolled from May 2017 to July 2020. Inclusion criteria considered BRVO of at least 3 months. All enrolled patients had a BCVA of 4.6 or worse,and a central macular thickness (CMT) of more than $250 \mu \mathrm{m}$, followed up for at least 9 months after the first IVC, had recurrent ME. The recurrent ME are the cases in which the CMT still above $250 \mu \mathrm{m}$ after the IVC for three times. Patients were excluded if they met the following criteria: pregnancy, had uncontrolled hypertension or diabetes, BRVO with macular ischemia, had sensitivity to sodium fluorescein, had received prior anti-VEGF injection or triamcinolone acetonide (TA) injection, had undergone scatter laser photocoagulation or ocular surgery 3 months before the event, or eyes with other ocular diseases.

\section{Treatment Methods}

All enrolled patients have been received intravitreal injection of conbercept (IVC) $0.5 \mathrm{mg}$ once every four weeks for 3 times. The patients received fluorescein angiography (FA) within 2 weeks after the third injection. Grid photocoagulation to the leaking area seen on the FA was performed when the leaking area was visible on the FA (The laser parameters are as follows: spot diameter, 100-200 $\mu \mathrm{m}$; power, 150$200 \mathrm{~mW}$; exposure time, $0.1 \mathrm{sec}$ ). But we could not obtain informed consent about grid laser photocoagulation from all patients. So IVC mono-therapy for patients who refuse grid laser photocoagulation therapy.

During follow-up period, all patients whose visual acuity was worse than 0.4 or central subfoveal thickness $>250 \mu \mathrm{m}$ would receive re-injection of conbercept. All operations were performed by the same physician.

\section{Efficacy And Safety Assessments}

Our primary objective was to evaluate the change in BCVA and CMT in both groups after treatment. The examination at 1 month, 3 month, and 6 month after 3 times IVC treatment was recorded. All patients 
with recurrent ME received re-injection after 3 times IVC treatment, and they had been followed up at month 1 after the retreatment. The BCVA was converted to a logarithm of the minimum angle of resolution (logMAR) for calculation. CMT was measured by optical coherence tomography (OCT). Foveal avascular zone (FAZ) and peripheral ischemia were assessed assessed by fluorescein angiography (FA) examinations at baseline and after the third injection.

\section{Statistical analysis}

Statistical analyses were performed using SPSS version 25.0 (http://www.spss.com). All values are expressed as mean \pm SD or proportions. Mann-Whitney test was adopted for the comparison of BCVA and CMT. A p-value of $<0.05$ was considered to be statistically significant.

\section{Results}

Demographics and clinical data are presented in Table 1. This study recruited 82 eyes of 82 patients (39 men and 43 women). All eyes showed recurrent macular edema associated with BRVO.The age (mean standard deviation) of the patients was $70.2 \pm 9.3$ years. Approximately $62 \%$ (51 patients) of affected eyes were complicated with hypertension, $21 \%$ (17 patients) suffered from diabetes. 30 ( $37 \%$ ) of the 82 patients had a history of smoking. 9 eyes (11\%) were Hemispheric Retinal Vein Occlusion (HSRVO). 35 eyes did not receive laser treatment during the follow-up period. No differences were observed in age, sex, baseline BCVA, baseline CMT, disease duration between the two groups $(P>0.05)$. The mean follow-up time of two groups were $10.9 \pm 1.9$ and $11.3 \pm 2.1$ months. The average number of injections was 4.7 in all patients (range 4-7). There are no significant difference was observed between the two groups $(P>$ 0.05). But we noted IVC + Laser group received fewer injections in HSRVO eyes $(p=0.036)$. 
Demography and baseline characteristics

\begin{tabular}{|llll|}
\hline & IVC & IVC + Laser & P-value \\
\hline no. & 35 & 47 & \\
\hline sex F/M & $21 / 14$ & $24 / 23$ & 0.424 \\
\hline age & $68.7 \pm 10.0$ & $71.3 \pm 8.7$ & 0.208 \\
\hline duration,m & $1.77 \pm 0.81$ & $1.79 \pm 0.75$ & 0.864 \\
\hline Hypertension,No. & 20 & 31 & 0.418 \\
\hline Diabetes mellitus,No. & 7 & 10 & 0.889 \\
\hline BCVA log MAR & $0.85 \pm 0.38$ & $0.89 \pm 0.43$ & 0.809 \\
\hline CMT $\mu m$ & $529.9 \pm 115.8$ & $545.8 \pm 107.2$ & 0.439 \\
\hline HSRVO & 4 & 5 & 0.91 \\
\hline Follow-up & $10.9 \pm 1.9$ & $11.3 \pm 2.1$ & 0.908 \\
\hline Number of IVC after 3 times in 9 m (BRVO) & $1.69 \pm 0.76$ & $1.74 \pm 0.85$ & 0.858 \\
\hline Number of IVC after 3 times in 9 m (HSRVO) & $2.5 \pm 0.6$ & $1.4 \pm 0.5$ & 0.036 \\
\hline $\begin{array}{l}\text { Abbreviations: BCVA: best-corrected visual acuity; logMAR: logarithm of the minimal angle of } \\
\text { resolution; CMT: central macular thickness; }\end{array}$ & & & \\
\hline
\end{tabular}

We compared BCVA and CMT between the two groups (Table 2). After 1 month, we noted a significant gain in BCVA in both groups(Fig. 1). During the follow-up period, we found a significant differences in $B C V A$ at 6 months versus baseline $(P<0.01)$. But in comparing the two groups, there are no significant differences in BCVA improvement at 1 month to 6 month after treatment between the two groups $(P>$ 0.05). ME obviously subsided after treatment in two groups (Fig. 2). During the follow-up period of 1 to 6 months, the changes of CMT in both groups had statistically significant differences between baseline and after treatment $(P<0.01)$ (Fig. 3). But at 1 month to 6 month after treatment, there are no significant difference was observed between the two groups $(P>0.05)$. 
Table 2

Comparison of outcomes of the studied patients in two different BRVO groups

\begin{tabular}{|c|c|c|c|c|c|}
\hline & & baseline & $1 \mathrm{~m}$ & $3 \mathrm{~m}$ & $6 \mathrm{~m}$ \\
\hline \multirow[t]{3}{*}{ BCVA(BRVO) } & ivc & $0.85 \pm 0.38$ & 0.54 & 0.31 & 0.29 \\
\hline & ivc + laser & $0.89 \pm 0.43$ & 0.53 & 0.3 & 0.26 \\
\hline & $\mathrm{p}$ & $0.809 *$ & $0.525^{\star}$ & $0.111^{*}$ & $0.239 *$ \\
\hline \multirow[t]{3}{*}{ CMT(BRVO) } & ivc & $529.9 \pm 115.8$ & 362 & 299.5 & 279.5 \\
\hline & ivc + laser & $545.8 \pm 107.2$ & 350.1 & 284.3 & 266.5 \\
\hline & $\mathrm{p}$ & $0.439 *$ & $0.287 *$ & $0.072^{*}$ & $0.11^{*}$ \\
\hline
\end{tabular}

We also compared HSRVO between the two groups (Table 3). There are also no significant difference was observed between the two groups $(P>0.05)$.

Table 3

Comparison of outcomes of the studied patients in two different HSRVO groups

\begin{tabular}{|c|c|c|c|c|c|}
\hline & & baseline & $1 \mathrm{~m}$ & $3 \mathrm{~m}$ & $6 \mathrm{~m}$ \\
\hline \multirow[t]{3}{*}{ BCVA(HSRVO) } & ivc & $0.86 \pm 0.29$ & $0.63 \pm 0.26$ & $0.38 \pm 0.10$ & $0.3 \pm 0.14$ \\
\hline & ivc + laser & $1.08 \pm 0.25$ & $0.54 \pm 0.15$ & $0.28 \pm 0.13$ & $0.26 \pm 0.11$ \\
\hline & $\mathrm{p}$ & $0.138^{*}$ & $0.9 *$ & $0.161^{\star}$ & $0.8^{\star}$ \\
\hline \multirow[t]{3}{*}{ CMT(HSRVO) } & ivc & $559.5 \pm 90.5$ & $384.8 \pm 69.9$ & $325.5 \pm 38.8$ & $279.8 \pm 48.7$ \\
\hline & ivc + laser & $612.4 \pm 109.6$ & $370.6 \pm 51.7$ & $277.0 \pm 26.4$ & $266.8 \pm 30.4$ \\
\hline & $p$ & $0.462 *$ & $0.9 *$ & $0.086^{\star}$ & $0.9 *$ \\
\hline
\end{tabular}

During follow-up time, no systemic or ocular complications (such as thromboembolism, intraocular hypertension, iatrogenic cataract, vitreous hemorrhage, retinal detachment and endophthalmitis) occurred.

\section{Discussion}

Complications of BRVO include macular edema, retinal neovascularization, macroaneurysmal formation, maculopathy, retinal telangiectasia, retinal detachment, and vitreous hemorrhage[12]. ME is the major cause of vision impairment and metamorphopsia. The main purpose of all treatments is the resolution of 
the macular edema to maintain central visual acuity $[13,14]$. It has been found that the level of VEGF in the vitreous body of BRVO eyes is higher than normal eyes, which may leads to neovascularization and ME. VEGF is a vascular permeability factor that plays a pivotal role in neovascularization. Conbercept can inhibit VEGF and placental growth factor competitively, inhibit angiogenesis and treating ME[15]. Intravitreal injections of anti-VEGF have become first-line treatment for symptomatic ME from BRVO. Many studies have reported that early Intravitreal injections treatment can improve visual outcome[16]. In our study, IVC therapy and IVC combined with Laser therapy significantly improved mean VA and reduced the mean CMT for recurrent ME secondary to BRVO. The combination treatment had a significant effect on reducing CMT and improving BCVA. But compared with IVC, it does not show great advantages. A prospective clinical trial indicated fixed monthly IVC over 3 months demonstrated a generally favorable safety and tolerability profile in the treatment of macular edema due to BRVO[8].

However, recurrence may occur if the IVC treatment has stopped. IVC therapy need multiple injections over periods of months to years. More importantly, multiple injections may causes some adverse effects. Such as endophthalmitis, cataract, retinal detachment, and intraocular hemorrhage. In addition, although IVC therapy is much cheaper than other anti-VEGF, it also has brought a certain economic burden for many patients.

Currently, laser photocoagulation is used for ME in patients with BRVO who are adverse reactions to intravitreal therapy or unable to pay high medical costs. Laser photocoagulation is a noninvasive and conventional treatment for BRVO. The research results about effectiveness of laser therapy for BRVO are different. Branch Vein Occlusion Study confirmed the efficacy of grid laser photocoagulation for treating macular edema due to BRVO in 1984[2]. More than a decade after that, this method become the standard treatment for BRVO. A review published in Cochrane included five studies conducted in Europe and North America. Among the five studies, one RCT with moderate-quality evidence supports the use of grid laser photocoagulation to treat macular edema following BRVO, and other evidence show intravitreal antivascular endothelial growth factor (VEGF) is not better than macular grid laser photocoagulation in $B R V O[17]$. A randomized clinical trial demonstrated that grid laser treatment is helpful in improving visual acuity in eyes with $\mathrm{ME}[18]$,but the results achieved are modest. Furthermore, one study on murine retina showed that VEGF protein level is found to increase a few days after laser treatment and then start to decrease in mouse eyes[19].So the laser treatment seems did not benefit in BCVA and resolution of edema. Most of the current studies still support vitreous injection. This may be due to that it is difficult to perform effective coagulation when macular edema is severe. But IVC treatment reduced the CMT allowed us to perform grid photocoagulation more safely. In our study, combination treatment reduced foveal thickness effectively, but there is no obvious advantage than IVC therapy. Considering the potential side effects of laser photocoagulation, we suggest that IVC without additional treatment be used for recurrent macular edema.

In this study, IVC alone and the combination therapy was performed to macular edema recurred.In the 9month followed up period, $52.4 \%$ of patients need $\geq 5$ injections. There was no significant difference between the two groups. Depending on the site of the arteriovenous crossing, BRVO was divided into 3 
main groups: major BRVO, HSRVO, and macular BRVO[12]. There was no macular BRVO has been found in our study. But we observed the treatment of HSRVO, number of IVC treatments in combination group is fewer than treatment with IVC alone. HSRVO is characterized by an occlusive process involving a hemiretina. The Central Vein Occlusion Study Group reported that grid photocoagulation can reduce CMT in eyes with RVO. Based on the current findings, laser photocoagulation seems has the auxiliary effect of reducing the macular edema associated with HSRVO. However, it is difficult to draw reliable conclusions because the number of cases is too small. Previous studies about intravitreal injection of bevacizumab have shown that additional grid laser photocoagulation has a substantial effect on reducing recurrent macular edema[20]. Based on the current results, we consider that IVC with or without retinal photocoagulation can effectively improve the visual acuity and reduce the CMT of patients. And the effect of this combination therapy on visual recovery and reduce the CMT is limited. But when it is difficult to perform repeated IVC or patients with financial difficulties, we can consider grid photocoagulation combined with IVC therapy.

There are a few limitations to this study. First, this was a retrospective study and small sample size. Then, we did not have a control group of patients who did not undergo any treatment. Furthermore, the followup duration is relatively short. The results in this study need to be further verified using large number of samples by a multicenter randomized clinical trial.

In conclusion, conbercept with or without retinal photocoagulation can effectively improve the visual acuity and reduce the CMT. We only suggest that combination therapy be used for recurrent macular edema associated with HSRVO or when it is difficult to perform repeated IVC.

\section{Declarations}

\section{Declarations}

\section{Ethics approval and consent to participate}

This study was approved by the Ethics Committee of Dongyang people's Hospital (Dongyang, China). Signed informed consents were obtained from all participants before the study.

\section{Consent for publication}

Not applicable.

\section{Availability of data and materials}

All data generated or analyzed during this study are included in this published article.

\section{Funding}

Not applicable. 


\section{Competing interests}

The authors declare they have no competing interests.

\section{Authors' contributions}

TGM and JYH designed the study and performed the experiments, TGM collected the data, TGM and HYS analyzed the data, TGM and WDW prepared the manuscript. All authors read and approved the final manuscript. Ethics approval and consent to participate.

\section{Author details}

Dongyang People's Hospital, Dongyang, 322100, Zhejiang Province, China.

\section{References}

1. Ho M, Liu DT, Lam DS, Jonas JB. Retinal Vein Occlusions, from Basics to the Latest Treatment. Retina. 2016;36(3):432-48.

2. Argon laser photocoagulation for macular edema in branch vein occlusion. The Branch Vein Occlusion Study Group. Am J Ophthalmol 1984, 98(3):271-282.

3. Jaulim A, Ahmed B, Khanam T, Chatziralli IP. Branch retinal vein occlusion: epidemiology, pathogenesis, risk factors, clinical features, diagnosis, and complications. An update of the literature. Retina. 2013;33(5):901-10.

4. Braithwaite T, Nanji AA, Greenberg PB. Anti-vascular endothelial growth factor for macular edema secondary to central retinal vein occlusion. Cochrane Database Syst Rev 2010(10):CD 007325.

5. Liu W, Li Y, Cao R, Bai Z, Liu W. A systematic review and meta-analysis to compare the efficacy of conbercept with ranibizumab in patients with macular edema secondary to retinal vein occlusion. Medicine. 2020;99(21):e20222.

6. Qiao G, Dong WJ, Dai Y, Jiang ZH, Guo HK. Diabetic macular edema in proliferative stage treated with anti-vascular endothelial growth factor agent and triamcinolone acetonide by laser-based strategies. Int J Ophthalmol. 2017;10(7):1113-9.

7. Zhang M, Zhang J, Yan M, Luo D, Zhu W, Kaiser PK, Yu DC, Group KHPS. A phase 1 study of KH902, a vascular endothelial growth factor receptor decoy, for exudative age-related macular degeneration. Ophthalmology. 2011;118(4):672-8.

8. Sun Z, Zhou H, Lin B, Jiao X, Luo Y, Zhang F, Tao S, Wu Q, Ke Z, Liu X. Efficacy and Safety of Intravitreal Conbercept Injections in Macular Edema Secondary to Retinal Vein Occlusion. Retina. 2017;37(9):1723-30.

9. Li F, Sun M, Guo J, Ma A, Zhao B. Comparison of Conbercept with Ranibizumab for the Treatment of Macular Edema Secondary to Branch Retinal Vein Occlusion. Curr Eye Res. 2017;42(8):1174-8. 
10. Luo W, Jia F, Liu M, Wang Y, Zhang T. The Analysis of Correlative Factors of Visual Acuity with Intravitreal Conbercept Injection in Macular Edema Associated with Branch Retinal Vein Occlusion. J Ophthalmol. 2018;2018:7348153.

11. Spaide RF. RETINAL VASCULAR CYSTOID MACULAR EDEMA: Review and New Theory. Retina. 2016;36(10):1823-42.

12. Parodi MB, Bandello F. Branch retinal vein occlusion: classification and treatment. Ophthalmologica. 2009;223(5):298-305.

13. Noma H, Funatsu H, Harino S, Nagaoka T, Mimura T, Hori S. Influence of macular microcirculation and retinal thickness on visual acuity in patients with branch retinal vein occlusion and macular edema. Jpn J Ophthalmol. 2010;54(5):430-4.

14. Murakami T, Okamoto F, lida M, Sugiura Y, Okamoto Y, Hiraoka T, Oshika T. Relationship between metamorphopsia and foveal microstructure in patients with branch retinal vein occlusion and cystoid macular edema. Graefes Arch Clin Exp Ophthalmol. 2016;254(11):2191-6.

15. Xia JP, Wang S, Zhang JS. The anti-inflammatory and anti-oxidative effects of conbercept in treatment of macular edema secondary to retinal vein occlusion. Biochem Biophys Res Commun. 2019;508(4):1264-70.

16. Ehlers JP, Kim SJ, Yeh S, Thorne JE, Mruthyunjaya P, Schoenberger SD, Bakri SJ. Therapies for Macular Edema Associated with Branch Retinal Vein Occlusion: A Report by the American Academy of Ophthalmology. Ophthalmology. 2017;124(9):1412-23.

17. Lam FC, Chia SN, Lee RM. Macular grid laser photocoagulation for branch retinal vein occlusion. Cochrane Database Syst Rev 2015(5):CD008732.

18. Parodi MB, Spasse S, lacono P, Di Stefano G, Canziani T, Ravalico G. Subthreshold grid laser treatment of macular edema secondary to branch retinal vein occlusion with micropulse infrared (810 nanometer) diode laser. Ophthalmology. 2006;113(12):2237-42.

19. Konac E, Sonmez K, Bahcelioglu M, Kaplanoglu GT, Varol N, Sarac GN, Ozcan PY. Does Pattern Scan Laser (PASCAL) photocoagulation really induce less VEGF expression in murine retina than conventional laser treatment? Gene. 2014;549(1):156-60.

20. Ogino K, Tsujikawa A, Murakami T, Muraoka Y, Kurashige Y, Yoshimura N. Grid photocoagulation combined with intravitreal bevacizumab for recurrent macular edema associated with retinal vein occlusion. Clin Ophthalmol. 2011;5:1031-6.

\section{Figures}




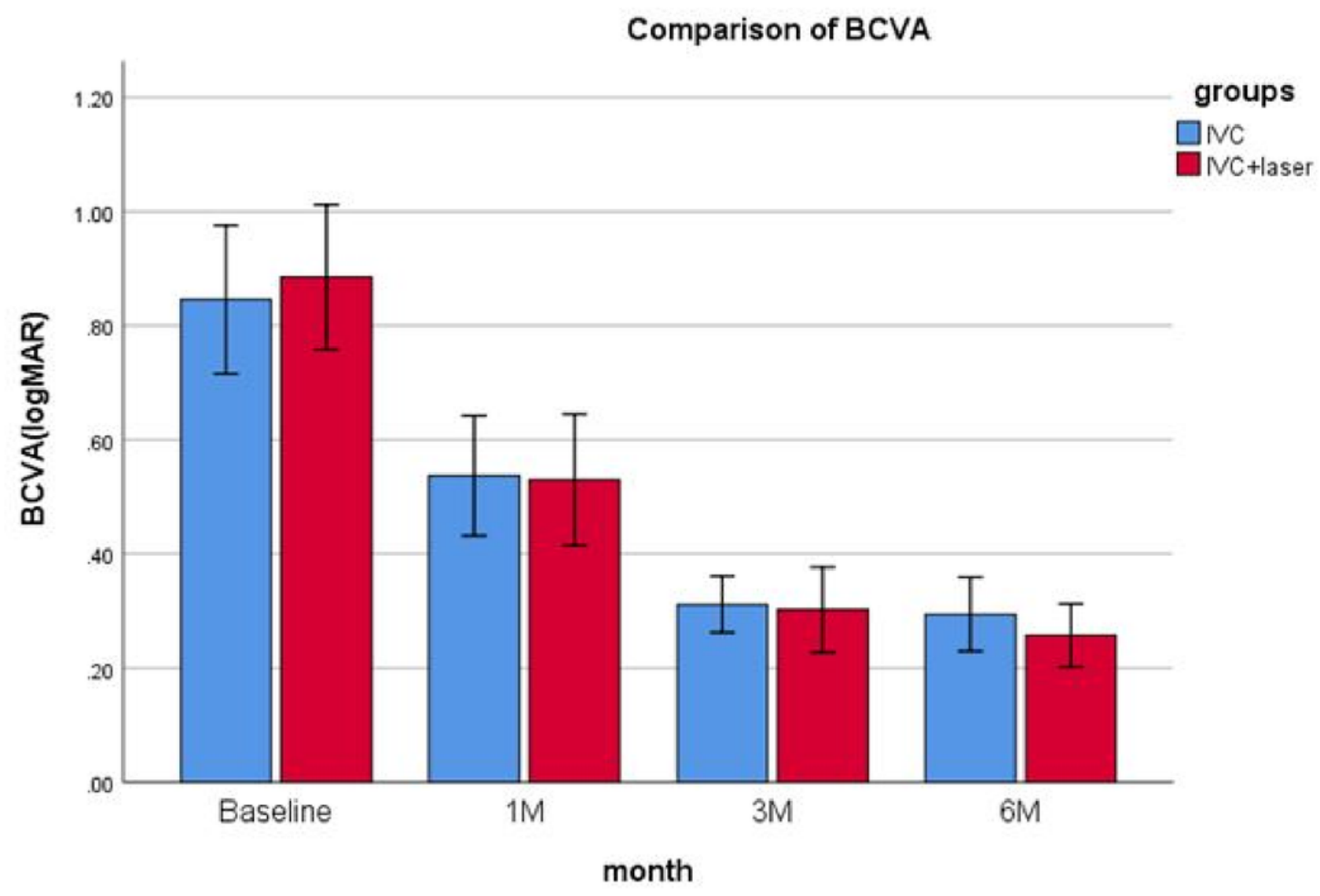

Figure 1

Comparison of pretreatment and post-treatment BCVA (logMAR) of the studied patients in two different groups. BCVA, best corrected visual acuity; logMAR, logarithm of the minimum angle of resolution. 


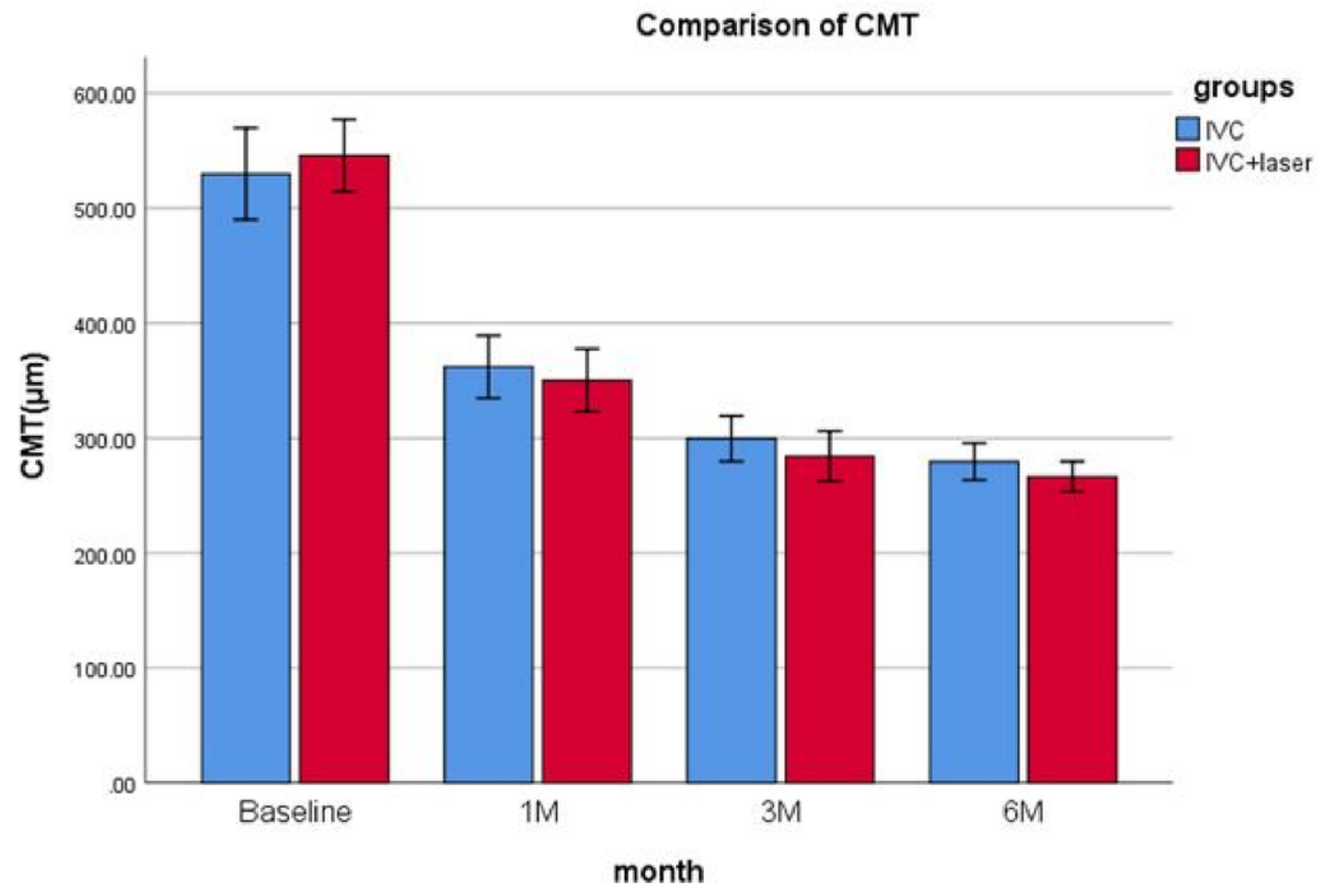

Figure 2

Comparison of pretreatment and post-treatment CMT $(\mu \mathrm{m})$ of the studied patients in two different groups. CMT, central macular thickness. 


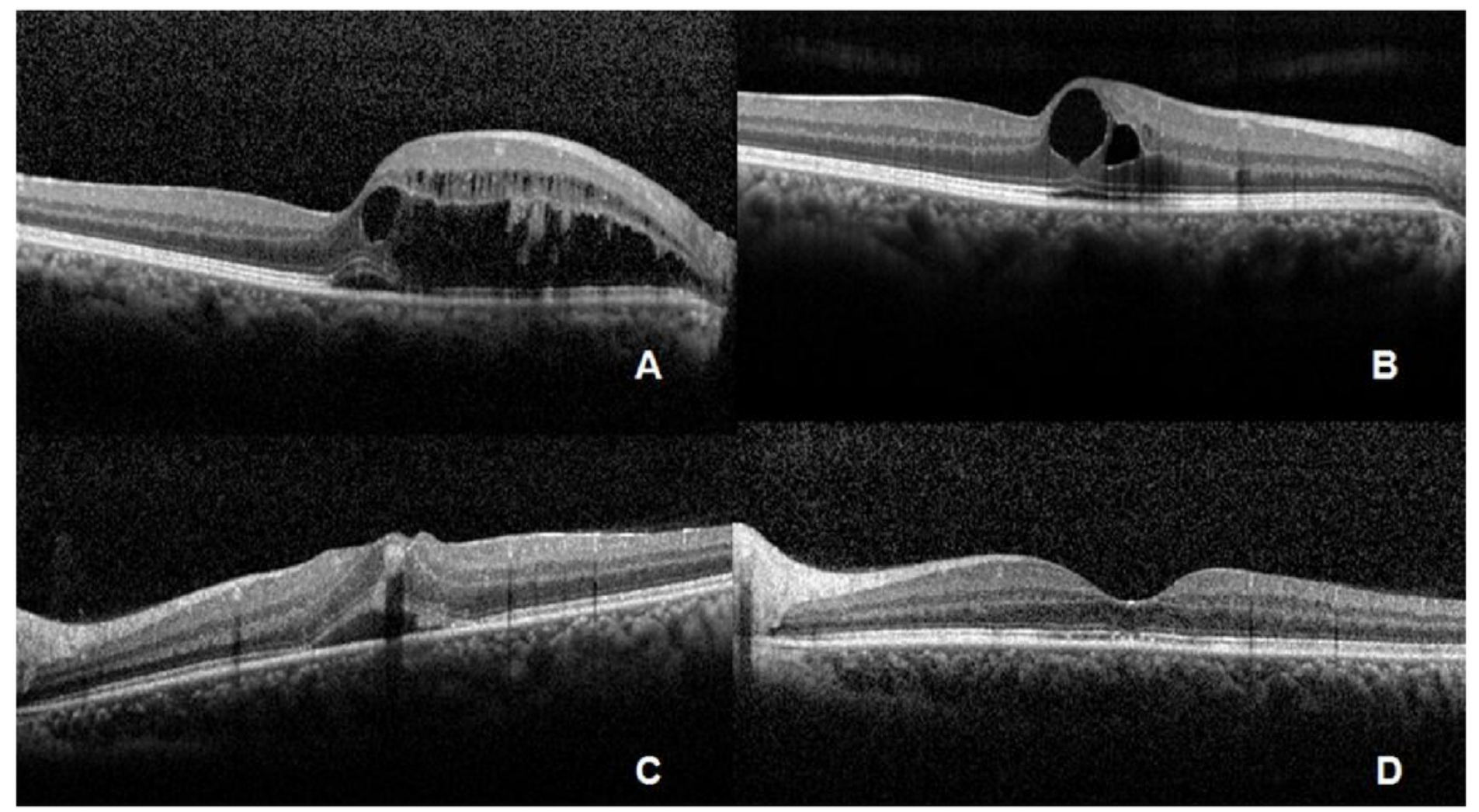

\section{Figure 3}

Intravitreal injection of conbercept (IVC) with or without retinal photocoagulation for recurrent macular edema associated with branch retinal vein occlusion. An 56-year-old man had a visual impairment in the right eye due to macular edema associated with branch retinal vein occlusion. (A).Optical coherence tomography (OCT) showed a recurrent macular cystoid edema at baseline. (B).OCT showed macular edema dramatically subsided at 6 month after combined therapy. An 75-year-old man had a visual impairment in the left eye due to macular edema associated with branch retinal vein occlusion. (C).OCT showed a recurrent cystoid space beneath the fovea at baseline. (D).OCT showed retinal structure almost recovered to normal at 6 month after IVC. 